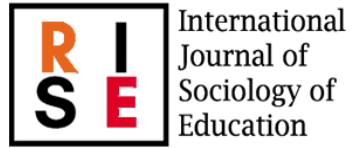

International

Journal of Sociology of Education

\section{Hipatia Press}

www.hipatiapress.com

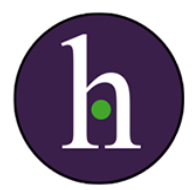

Instructions for authors, subscriptions and further details:

http://rise.hipatiapress.com

\title{
Violencia de Género Adolescente en Espacios Educativos: Nuevas Miradas desde la Región de La Araucanía, Chile
}

Juan Carlos Peña Axt ${ }^{1}$

Eduardo Sandoval-Obando ${ }^{1}$

1) Universidad Autónoma de Chile, Chile

Date of publication: February $25^{\text {th }}, 2021$

Edition period: February 2021-June 2021

To cite this article: Peña Axt, J.C., \& Sandoval-Obando, E. (2021).

Violencia de Género Adolescente en Espacios Educativos: Nuevas Miradas desde la Región de La Araucanía, Chile. International Journal of Sociology of Education, 10(1), 57-81. http://doi.org/10.17583/rise.2021.6090

To link this article: http://dx.doi.org/10.17583/rise.2021.6090

PLEASE SCROLL DOWN FOR ARTICLE

The terms and conditions of use are related to the Open Journal System and to Creative Commons Attribution License (CC-BY) 


\section{Adolescent Gender Violence in Educational Spaces: New Looks from the Araucanía Region, Chile}

Juan Carlos Peña Axt

Universidad Autónoma de Chile
Eduardo Sandoval-Obando

Universidad Autónoma de Chile

(Received: 3 July 2020; Accepted: 2 October 2020; Published: 25 February 2021)

\section{Abstract}

This study is a quantitative investigation that was oriented to know the manifestations of violence in adolescent relationships in educational establishments in the city of Temuco, Chile. The sample was 471 high school students of which 262 are women (55.6\%) and 209 men (44.4\%). The results show two main lines, the first of which is the bidirectionality of violence in adolescent relationships, present in all types of violence analyzed. Secondly, higher percentages of violence suffered by men are established in many of the variables, which is explained through the socialization of masculinity. The results of this point project the need to continue studying masculinities at an early age to transform the effective relationships that are being created among adolescents.

Keywords: violence, students, courtship, gender, masculinities 


\section{Violencia de Género}

\section{Adolescente en Espacios} Educativos: Nuevas Miradas

\section{desde la Región de La Araucanía, Chile}

Juan Carlos Peña Axt

Universidad Autónoma de Chile
Eduardo Sandoval-Obando

Universidad Autónoma de Chile

(Recibido: 3 Julio 2020; Aceptado: 2 Octubre 2020; Publicado: 25 Febrero 2021)

\section{Resumen}

Este estudio es una investigación cuantitativa que estuvo orientada a conocer las manifestaciones de violencia en las relaciones adolescentes en establecimientos educacionales de la ciudad de Temuco, Chile. La muestra fue 471 estudiantes de secundaria de los cuales 262 son mujeres (55.6\%) y 209 hombres (44.4\%). Los resultados evidencian dos líneas principales, la primera de ellas es la bidireccionalidad de la violencia en las relaciones de pareja adolescentes, presente en todos los tipos de violencia analizados. En segundo lugar, se establecen mayores porcentajes de violencia sufrida por hombres en gran parte de las variables lo que se explica a través de la socialización de la masculinidad. Los resultados de este punto proyectan la necesidad de continuar con el estudio de las masculinidades en edades tempranas para transformar las relaciones efectivas que se están creando entre los adolescentes.

Palabras clave: violencia, estudiantes, noviazgo, género, masculinidades

2021 Hipatia Press

ISSN: 2014-3575

http://doi.org/10.17583/rise.2021.6090
Hipatia Press www.hipatiapress.com

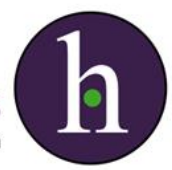




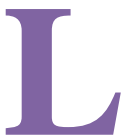

a violencia de género en las relaciones afectivas sexuales en edades tempranas ocupa un lugar relevante en las investigaciones a nivel nacional e internacional (Albertson, Moreno, Garrison, Evans y Ahrens, 2018), debido principalmente a que la preocupación estuvo centrada durante muchos años en la violencia intrafamiliar, descuidando la violencia en edades tempranas (Bittar y Nakano, 2017; Peña, Arias y Boll, 2019). En Chile, hasta hace muy poco no existía una ley que reconociera las relaciones de parejas de novios, esto ha supuesto una invisibilización en lo investigativo (Bittar y Nakano, 2017) lo que finalmente acaba por esconder una problemática urgente. Uno de los elementos claves de la investigación de la violencia en la adolescencia, es sin duda las consecuencias en la vida adulta (Epstein et al., 2018; Kahn y Halpern, 2018), no sólo en lo emocional o social, sino que generaría consecuencias al corto, mediano y largo plazo sobre la salud mental de las víctimas (Aguila et al., 2016). Lo anterior, sería explicable en parte por la reproducción de un sistema escolar patriarcal que valida la distribución desigual del capital cultural (Gómez, 2017), la cosificación de lo femenino y la perpetuación de la violencia simbólica en contra de la mujer (Benería, 1981; Sandoval-Obando, 2018; Sandoval y Peña, 2019).

El presente artículo está conformado por cuatro apartados. En primer lugar, se realiza una introducción a la temática en estudio, así como los elementos teóricos que permiten contextualizar en el plano local el objeto y campo en el que se desarrolló la presente investigación. En segundo lugar, se describen los aspectos metodológicos que orientaron este trabajo, señalando el diseño, las características de los participantes, así como el instrumento que se empleó para la recolección y posterior análisis de los datos. En tercer lugar, se presentan los resultados generales que emergieron de esta investigación dando cuenta de las principales dimensiones: violencia psicológica, violencia en Redes Sociales (RRSS), violencia física y violencia sexual. En cuarto lugar, se realiza una discusión en torno a los resultados obtenidos y cómo estos se relacionan con la literatura disponible en torno a las principales categorías analizadas: la bidireccionalidad y la masculinidad, como elementos determinantes en la violencia de género en adolescentes. Por último, en las conclusiones se señalan en tres grandes ejes que emergen como proyecciones de esta investigación, para seguir explorando y comprendiendo las relaciones afectivas que establecen los adolescentes. 


\section{Peña Axt \& Sandoval Obando - Violencia de Género}

\section{Contextualización Teórica del Estudio}

En Chile, el estudio de la violencia en la adolescencia comienza con Aguirre y García (1997) orientado a población universitaria. En dicho trabajo, establecen que el tipo de violencia más común en la adolescencia es la psicológica. Así, el $51 \%$ de los encuestados señala haber sufrido violencia psicológica dentro de su relación de pareja. Los estudios más recientes están a cargo del Instituto de Nacional de la Juventud (INJUV). Específicamente, en el Cuarto Sondeo de Violencia en el Pololeo (INJUV, 2016) encontraron que los celos se constituyen como la mayor causa de situaciones de violencia entre las parejas (49\%) en jóvenes de 15 a 29 años. Un estudio realizado en estudiantes universitarios en Chile señala que el tipo de violencia más recurrente es la psicológica con un $18 \%$ de prevalencia (Sandoval y Peña, 2019). En la región de La Araucanía, Vizcarra y Póo (2011) señalan que el $57 \%$ de los encuestados dice haber sido víctima de maltratos psicológicos. La violencia de carácter psicológica ha encontrado una forma puntual de manifestación a través de las redes sociales. Éstas son un medio de comunicación importante en las relaciones de los adolescentes (Da Silva, 2016; Linne y Angilletta, 2016; Peña et al., 2019). El INJUV (2016) ha identificado a Facebook, Youtube y Twitter como las redes sociales en las cuales se manifiesta la violencia dentro de las relaciones afectivas en adolescentes (64\%). En este sentido, Vázquez y Pastor (2019) señalan que la mensajería instantánea y las redes sociales son una herramienta muy importarte de comunicación y además de conflictos en las relaciones afectivosexuales en los adolescentes (Mena-Rodríguez y Velasco-Martínez, 2017). Los resultados apuntan a que mientras mayor es el uso de las redes y la mensajería instantánea, mayores posibilidades de que haya conflictos (Vázquez y Pastor, 2019). Un estudio realizado por Borrajo y Gámez-Guadix (2016) revelan que la principal forma en la cual se manifiesta la violencia en las relaciones adolescentes en las redes sociales es a través del control con una prevalencia de un $81 \%$. Este promedio indica que la prevalencia es muy alta en ambos sexos, siendo de un $80.4 \%$ para mujeres y de un $82.8 \%$ para hombres. Un elemento importante, está relacionado con las consecuencias de este tipo de violencia puesto que está muy ligada a la depresión en los jóvenes. 
Otro estudio realizado por García et. al. (2017) señala que los hombres presentaron una mayor prevalencia de ciber-violencia en la pareja tanto como víctimas y como agresores. Un estudio relevante realizado Martín, et al. (2016) señalan que las redes sociales se han constituido como una herramienta para realizar control, usurpar la personalidad e incluso violar la intimidad en caso de rupturas (Martín et. al., 2016).

En cuanto a la violencia física en Chile, estudios vinculados a la temática señalan que un $6.3 \%$ de jóvenes han recibido violencia física en su relación de pareja, de la cual un $6.5 \%$ corresponde a mujeres y un $6 \%$ a varones en Chile (Octava Encuesta Nacional de Juventud, INJUV, 2017). Una investigación realizada en la ciudad de Osorno con población universitaria determinó que el $23.1 \%$ de los jóvenes ha sufrido violencia física, donde el $27.2 \%$ fue para las mujeres y un $16.2 \%$ de hombres vivenciaron situaciones de este tipo en manos de sus parejas. En lo específico, este estudio dice relación con quienes han ejercido la violencia, específicamente, evidenciando que son las mujeres quienes mayormente ejercen la violencia física con un $30.4 \%$, frente a un $12.5 \%$ en hombre (Vivanco et al, 2015). Bajo la misma línea, Saldivia y Vizcarra desarrollaron en 2012 una investigación en donde el $31.7 \%$ de los encuestados experimentaron violencia física dentro de su relación de pareja. En cuanto a la distinción entre víctima y agresor, este mismo estudio señala en cuanto a la violencia física, un $16.1 \%$ señala ser víctima, y un $14.6 \%$ agresor(a) (Saldivia y Vizcarra, 2012). Vizcarra y Póo (2011), por su parte sostienen que el $26 \%$ de los estudiantes que participaron en su estudio manifiestan haber experimentado situaciones de violencia física por parte de sus parejas (Vizcarra y Póo, 2011).

En lo que refiere a la violencia sexual Vivanco et al. (2015) sostienen que las mujeres son quienes sufren mayoritariamente este tipo de violencia, así se establece una prevalencia de un $8.8 \%$ para hombres y de un $16.1 \%$ en mujeres (Vivanco et al., 2015). El estudio desarrollado por Schuster et al (2016) vinculado a la prevalencia de violencia sexual entre estudiantes universitarios reveló que el $26.3 \%$ correspondiente a mujeres y un $27.2 \%$ de hombres declararon haber sido víctimas de agresión sexual. Bajo la misma línea nacional, el $17.1 \%$ de las mujeres y el $11.6 \%$ de hombres encuestados por Lehrer, Lehrer y Oyarzún (2009) refirieron haber sido víctimas de agresión sexual dentro de los últimos 12 meses. Los resultados de este trabajo sugieren 


\section{Peña Axt \& Sandoval Obando - Violencia de Género}

además que las situaciones vinculadas a "violación por un conocido" o "violación en la primera cita" son una problemática en la población estudiantil chilena.

La adolescencia es una etapa de la vida en donde los jóvenes comienzan a experimentar cambios importantes en sus vidas especialmente ligadas a sus vidas afectivo-sexuales (Díaz-Aguado, 2003; Dávila, 2004; Xóchitl, Sánchez y Robles, 2013; Rey-Anacona, 2013; Gaete, 2015). Este factor es sumamente relevante puesto que las investigaciones señalan que las mujeres adultas cuando hacen referencia al inicio de la violencia de la que han sido víctimas, señalan la etapa del noviazgo como la etapa de comienzo de la violencia que termina por ser habitual en la vida adulta (Kaufman-Parks, DeMaris, Giordano, Manning y Longmore, 2018; Gómez, Rodríguez y Quintanar, 2019). Es precisamente durante la adolescencia donde se consolidan y construyen valores ligados a las dinámicas afectivo-sexuales entre hombres y mujeres (Novo, Herbón y Amado, 2016; Peña et al., 2019), es en esta etapa vital en donde se construyen expectativas en cuanto a las relaciones, las que muchas veces configuran patrones relacionales violentos entre hombres y mujeres (Gómez et al., 2019).

En cuanto a la violencia suscitada durante la adolescencia, se podría señalar que esta posee las mismas características que la violencia experimentada en la adultez respecto a los tipos psicológica, física, sexual y económica. No obstante, algunos estudios señalan que la violencia física puede ser menos manifiesta o que existe una tendencia a ocultarla por el contexto en donde se desarrolla (Vizcarra y Poo, 2011; Mingo y Moreno, 2015). Por otro lado, la violencia económica es menos intensa principalmente porque no existe dependencia económica en este tipo de relaciones. Las mayores dificultades están, sin embargo, en el ámbito legal puesto que la ley mantenía hasta ahora, fuera este tipo de relaciones impidiendo un verdadero abordaje institucional, así como también investigativo puesto que ha sido una temática poco estudiada (Sanhueza, 2016; Bittar y Nakano, 2017; Valdivia et al., 2019). Dentro de las características propias de la violencia en las relaciones adolescentes, la literatura señala un elemento sumamente relevante que está vinculado con la bidireccionalidad (O’Leary y Slep, 2003; Vizcarra y Póo, 2011; Messinger, 2018). Este podría estar explicado por la simetría en cuanto a poder de este tipo de relaciones de pareja. Otro rasgo característico 
para explicar la violencia en las relaciones adolescentes está vinculado a los celos. Son éstos, los que provocan la mayor cantidad de conflictos y con ello violencia en las relaciones de adolescentes (Estébanez, 2012; Bair-Merritt y Matson, 2017; Peña et al., 2019).

Una de las particularidades que posee la violencia en las relaciones afectivas-sexuales de los adolescentes, está referida a la utilización de las redes sociales como principal forma de comunicación (Wright, 2020). En este sentido, muchos de los conflictos de los adolescentes se producen por interacciones que se establecen en las redes sociales o por interacciones de terceros en las mismas redes. Son precisamente las redes sociales una de las principales formas de comunicación de los jóvenes y adolescentes (Da Silva, 2016). Lo anterior, implica necesariamente que gran parte de las interacciones que existen en las relaciones de pareja por parte de los jóvenes, se producen de forma virtual. Estudios señalan que es en este espacio virtual donde se producen gran parte de las interacciones violentas en los adolescentes (Deans y Bhogal, 2019), y con ello aumenta la percepción de violencia en las redes sociales, considerando a éstas como espacios violentos y poco amigables (Blanco, 2014; Donoso, Rubio y Vilá, 2017).

La literatura explica como principal motivo la violencia en las relaciones de pareja a través de la socialización del género. Lo anterior, refleja que existen determinados modelos de socialización y atractivo en los que se instala y reproduce una socialización hacia la violencia y las desigualdades entre hombres y mujeres (Gómez, 2004; Duque, 2006; Padrós, 2012; Ríos, Peña, Duque y De Botton, 2018). Además, se genera una idealización asociada a estereotipos de género que determina las dinámicas relacionales y afectivas en adolescentes (Martínez-Marín y Martínez, 2019), influyendo por ejemplo en el control, siendo visto como una forma de preocupación (HernandoGómez, 2007; González-Ortega et al., 2008). Estas formas de socialización implicarían una bidireccionalidad de la violencia, puesto que el mayor equilibrio en estas edades no hace desaparecer la violencia, sino que por el contrario se agudizan (Villanueva-Blasco y Grau-Alberola, 2019). En la revisión del estado del arte, se observa que en las masculinidades se arraiga la violencia, según Flecha, Ríos y Puigvert, (2013), es en la socialización de la masculinidad donde se configuran las relaciones violentas. En primer lugar, encontramos las MTD (masculinidad tradicional dominante) cuando es el 
hombre quien ejerce la violencia y con ellos las desigualdades de género. En segundo lugar, tenemos las MTO (Masculinidad tradicional oprimida) que se entienden a hombres buenos pero subordinados. Es en este tipo de masculinidades que se da un tipo de violencia inversa, siendo la mujer la que ejerce el poder y en algunos casos la violencia. Este modelo no ayuda a superar las desigualdades de género ni la violencia puesto que se invierten los roles y la violencia continúa siendo una masculinidad complementaria de la MTD (Flecha, Puigvert y Ríos, 2013).

\section{Metodología}

\section{Tipo de Estudio}

Esta investigación es un estudio de carácter cuantitativo, centrada en estudiantes de educación secundaria de instituciones públicas (municipales) y concertadas (particulares subvencionados) de la ciudad de Temuco, Región de la Araucanía (Chile). La selección del tipo de metodología cuantitativa permite acceder a poblaciones grandes, 19.333 estudiantes de educación secundaria en la comuna de Temuco (Secretearía regional ministerial, SEREMI Araucanía, 2017).

\section{Participantes}

La muestra fue de tipo no probabilístico o intencionado (Canales, 2006) se conformó en base a los siguientes criterios: a) edad entre 14 a 18 años, b) estudiantes matriculados en las instituciones antes descritas, c) estar cursando la educación secundaria al momento de aplicar la encuesta, d) consentimiento informado aprobado por padres o tutores, y e) firma de asentimiento informado por parte de los estudiantes. La muestra quedó conformada por 471 estudiantes de secundaria de los cuales 262 son mujeres (55.6\%) y 209 hombres (44.4\%). Además, el $80 \%$ de los encuestados se encontraba en una relación de pareja al momento de responder el instrumento. Para acceder a los sujetos, se estableció contacto con seis establecimientos educacionales públicos y subvencionados de la ciudad de Temuco y se aplicaron consentimientos informados a estudiantes y padres en el caso de los 
adolescentes menores de 18 años.

\section{Instrumento}

El instrumento utilizado fue una encuesta auto aplicada, lo que permite asegurar la confidencialidad de las opiniones y experiencias de los estudiantes que participaron. El instrumento estuvo conformado por 37 preguntas orientadas a identificar los tipos de violencia en las relaciones de pareja adolescentes. Para la validación de este se utilizó la validación a través de jueces expertos. El instrumento además fue evaluado por el Comité de ética científico de la universidad.

\section{Análisis de la Información}

El análisis de la información se realizó a través de estadísticas descriptivas y tablas de frecuencias con el apoyo de la herramienta informática SPSS versión 23 para determinar los tipos de violencia, sexo y edad de quienes se manifiestan como víctimas de violencia. El análisis a través de estadísticas descriptivas se justifica puesto que permite un trabajo de difusión e intervención en prevención de charlas de violencia adolescente con las instituciones educacionales que acceden a participar del estudio.

\section{Resultados}

Los resultados del estudio están divididos en función de los tipos de violencia existentes en las relaciones adolescentes, organizándose en cuatro dimensiones. Para cada una de ellas, se elaboraron ítems (preguntas) orientadas a medir prevalencia de violencia en las relaciones afectivo-sexuales adolescentes. En primer lugar, se presentan los resultados de la violencia psicológica. En segundo lugar, lo relacionado con el control en las redes sociales. En tercer lugar, la violencia física y por último la violencia sexual. A continuación, se presentan los resultados más relevantes de cada uno de los tipos de violencia antes mencionados. 


\section{Peña Axt \& Sandoval Obando - Violencia de Género}

\section{Violencia Psicológica.}

Para explorar y determinar la presencia de violencia psicológica, se diseñaron cuatro ítems agrupados en la dimensión violencia psicológica, lo que permitió verificar las manifestaciones de violencia psicológica. En estos ítems, los resultados indican que un $77.8 \%$ de los adolescentes encuestados reconocen haber sido víctima de celos por parte de su pareja. Específicamente, las mujeres presentan un $81.7 \%$ de presencia en cuanto a los celos por parte de sus parejas, mientras que los hombres presentan un $73 \%$. Cuando los estudiantes fueron consulados sobre ¿tu pareja te ha criticado/burlado frente a otras personas? Los encuestados responden verse afectados en un $32.8 \%$. Por otra parte, los varones presentan mayor incidencia con un $37.5 \%$ en comparación al $29.1 \%$ por parte de las mujeres. Al ser consultados respecto si han sido víctimas de insultos, los encuestados reconocen haber sufrido insultos dentro de sus relaciones un $26.3 \%$ manifiesta haber sido víctima de esto, siendo separados por sexo en un $19.6 \%$ para mujeres y un $34.8 \%$ para los hombres, quienes nuevamente presentan mayor prevalencia.

Cuando los participantes fueron consultados sobre ¿tu pareja ha resaltado tus defectos en público o en privado? Los estudiantes manifiestan en un $24 \%$ ser víctima de este tipo de violencia. El porcentaje se presenta más alto para los varones (28.8\%), en comparación con las mujeres (20\%). Las situaciones en donde se compara de forma negativa a la pareja actual con exparejas, asociando esto a una expresión de violencia, los resultados destacan que un $14.7 \%$ de los encuestados afirma que fueron víctimas de esto. Separados por sexo, las mujeres presentan un porcentaje menor con $13.2 \%$, mientras que los hombres presentan un $16.5 \%$. Siendo éstos últimos quienes presentan mayor porcentaje de prevalencia.

$\mathrm{Al}$ analizar los resultados vinculados a violencia psicológica asociadas al control, éstos indican que, del total de la muestra, un $18.4 \%$ de los estudiantes reconoce que su pareja le ha pedido modificar algún aspecto de su vestimenta (falda, polera, pantalón, etc.), fundamentando que se viste provocativamente. Esta cifra posee mayor prevalencia en mujeres, donde se observa un $20.4 \%$ frente a un $16 \%$ en los hombres. 


\section{Control en las Redes Sociales}

De acuerdo con lo señalado precedentemente, se vislumbra como uno de los principales ámbitos de interacción entre jóvenes. Es decir, son las redes sociales el ámbito en donde se producen las principales interacciones de los adolescentes y estas redes sociales no están exentas de manifestaciones de violencia y control (Blanco, 2014; Da Silva, 2016; Donoso, Rubio y Vilá, 2018). Por ende, el porcentaje de jóvenes que posee redes sociales supera el 90\%, siendo un $98.8 \%$ de mujeres encuestadas y un $93.9 \%$ de hombres poseen redes sociales como Facebook, Whatsapp o Correo Electrónico. De acuerdo con los resultados de la encuesta, un $20.5 \%$ de los jóvenes señalan que les controlan Facebook, un $30.8 \%$ les controlan Whatsapp y un $4.3 \%$ les controlan el Correo Electrónico. Siendo Whatsapp la aplicación en donde hay mayor control por parte de las parejas adolescentes.

En conexión con lo anterior, el instrumento explora si la pareja de los participantes se enfadan o no, al ser contactado por otra persona de sexo opuesto en redes sociales. El estuduo encontró que el 52\% de las mujeres encuestadas dicen que sus parejas se enojan, mientras que un 54\% de los hombres encuestados dicen que sus parejas se enojan. En esta misma línea, al ser consultados si su pareja se enoja cuando los etiquetan en alguna publicación, nuevamente los hombres muestran un mayor porcentaje que las mujeres. Al respecto, los hombres afirman que su pareja se enoja en un 33.5\%, mientras que las mujeres afirman en un $27 \%$, siendo de un $30 \%$ para ambos sexos. Otro punto relevante obedece a la eliminación de cuentas en redes sociales. En estos casos, ocurre de forma inversa a lo que sucede en los casos anteriores ya que aquí las mujeres muestran una mayor porcentaje que los hombres. Así las mujeres dicen que sus parejas les han solicitado la eliminación de cuentas en Redes Sociales en un 14.6\%, mientras que los hombres manifestaban en un $13.5 \%$.

$\mathrm{Si}$ bien los casos anteriores muestran formas importantes de control y violencia en el uso de las redes Sociales, existen manifestaciones un poco más sutiles pero que de igual forma implican formas de violencia y control. Frente a esto, se establecieron dos preguntas ¿tu pareja obliga a tener datos y GPS 


\section{Peña Axt \& Sandoval Obando - Violencia de Género}

activados? y ¿tu pareja te obliga a activar horario de últimas conexiones? Estas dos manifestaciones de control si bien pueden ser menos violentas como otro tipo de violencias, sí se constituyen como un elemento perjudicial para las relaciones. Por ende, ante la primera pregunta de ¿tú pareja te obliga a tener los datos y GPS activados?, las mujeres manifiestan este tipo de comportamiento por parte de sus parejas en un $5.2 \%$, mientras que para los hombres esta pregunta marcó un $8.9 \%$, siendo para ambos sexos un $6.8 \%$ de prevalencia. Por otra parte, en lo referido a si tu pareja te ha obligado a activar la ultima hora de conexión, los datos indican que existe un $13 \%$ de prevalencia para este tipo de control. De esta forma las mujeres nuevamente muestran una menor prevalencia que los hombres con un 9.4\%, mientras que los varones muestran un $16.6 \%$ de prevalencia.

\section{Violencia Física}

Dentro de las variables asociadas a violencia física, el estudio exploró esta dimensión a través de tres ítems, observándose un promedio para ambos sexos de un $35.4 \%$. En este sentido, la primera pregunta estuvo orientada a conocer si ifrente a una discusión tu pareja te ha empujado o tomado con fuerza? Frente a esta situación, un $12 \%$ de los estudiantes de ambos sexos señalan haber vivenciado esta situación. En lo específico, las mujeres plantean que frente a esta situación un $9.6 \%$ de las encuestadas ha sufrido este tipo de violencia. Mientras que en el caso de los hombres encuestados un 14\% declara haber sido víctima de este tipo de violencia. Cuando los estudiantes fueron consultados si la pareja les ha lanzado objetos al discutir, nuevamente las mujeres plantean ser víctimas en menor medida que los hombres. Así, las mujeres muestran un $4 \%$ de prevalencia, frente a un $10 \%$ de los hombres con un total de un $7 \%$ para ambos sexos. Por otra parte, al ser consultados si las parejas les han causado lesiones físicas. Las mujeres muestran en un $4.4 \%$ haber sido víctimas der lesiones, frente a un $6.5 \%$ de los hombres respectivamente.

\section{Violencia Sexual.}

En lo global, los participantes muestran un $46 \%$ de violencia sexual para 
ambos sexos. Al ser consultados si han cedido ante los deseos sexuales de su pareja, un $41.7 \%$ de la muestra afirmaron haberlo hecho. Al ser separados por sexo un $30.7 \%$ de las mujeres encuestadas afirma haber cedido a los deseos de sus parejas. Por su parte, el $55.6 \%$ de los hombres encuestados afirma que ha cedido a los deseos de sus parejas. Al ser consultados por los acercamientos de carácter sexual (besos, caricias íntimas, etc.) un $75.8 \%$ afirma que ha sido porque ambos han querido. Al ser separados por sexo, el $5.5 \%$ de los hombres declara haber tenido acercamientos porque su pareja lo ha querido, frente a un $3.3 \%$ de las mujeres que manifiesta haber accedido porque sus parejas lo desean.

En esta misma dimensión, se elaboró un ítem sobre la responsabilidad de prevenir embarazos. Frente a esto, los participantes mostraron alta similitud en sus respuestas, planteando que es una responsabilidad compartida en un $89.9 \%$ por parte de las mujeres, frente a un $89.7 \%$ para los hombres promediando entre ambos sexos un $89.8 \%$. Al ser consultados si creen que la responsabilidad debe caer exclusivamente en la mujer, los encuestados sostienen en un $2.8 \%$ en promedio que debe ser una responsabilidad mayoritariamente de la mujer. Al ser separados por sexo, el 3.2\% de las mujeres cree que la responsabilidad es exclusiva de la mujer, frente a un $2.6 \%$ de los hombres que sostiene que la responsabilidad es exclusiva de la mujer. En cuanto a los que sostienen que la responsabilidad es exclusiva de los hombres, solo un $1.6 \%$ de las mujeres afirma que es responsabilidad de los hombres, en comparación con un $3.1 \%$ de los hombres que afirman que la prevención de embarazos es responsabilidad exclusivamente de los hombres.

\section{Discusión}

Este estudio estuvo enfocado en determinar los niveles de violencia de género en las relaciones adolescentes de estudiantes de secundarios residentes en la ciudad de Temuco, región de La Araucanía (Chile). De acuerdo con los datos recopilados, la violencia en sus distintos tipos de manifestaciones es una realidad presente en los estudiantes de las instituciones que participan de la investigación. Los resultados de este trabajo se pueden interpretar en dos dimensiones fundamentales: La primera, es la asociada a los tipos de violencia, bidireccionalidad presente en las relaciones de pareja en jóvenes. 


\section{Peña Axt \& Sandoval Obando - Violencia de Género}

La segunda dimensión está vinculada a las masculinidades. Estos elementos de interpretación de los resultados están relacionados entre sí, lo que impide hacer una lectura separada de los resultados.

Para comenzar se ha establecido que existe a una alta bidireccionalidad de la violencia en edades adolescentes (Mrug y Windle, 2009) siendo corroborado por los resultados de este estudio. Las investigaciones centradas en esta población son enfáticas en señalar que las relaciones de parejas esporádicas o no en edades tempranas, poseen un elevado porcentaje de prevalencia en violencia y bireccionalidad (O’Leary y Slep, 2003; Vizcarra y Póo, 2011; Sanhueza, 2016). Los niveles (\%) de los distintos tipos de violencia que se identificaron en las instituciones educacionales que fueron parte del estudio, varían según el tipo de violencia. Sin embargo, en todas las variables analizadas existe un porcentaje importante de bidireccionalidad, siendo sin duda la violencia psicológica la que presenta una mayor prevalencia con un $35 \%$. Esto es coincidente con los señalados por otras investigaciones en el ámbito nacional e internacional (Connolly y McIsaac, 2011; Adams y Williams, 2014; INJUV, 2016; Sandoval y Peña, 2019). De hecho, al ser analizada esta variable por ítem alcanza en el caso de los celos a un $78 \%$ de prevalencia para ambos sexos. Esto sin duda habla de una alta presencia de este tipo de violencia, porcentaje que es coincidente con otro estudio realizado en la región de La Araucanía (Chile) en la cual se establece una normalización de los celos como parte de las relaciones adolescentes (Peña et al., 2019).

Referente a las RRSS, existe una alta presencia del uso de estas aplicaciones por parte de los estudiantes que participaron en esta investigación, siendo coherente con lo encontrado en investigaciones previas (Linne y Angilletta, 2016; Peña et al. 2019; Vázquez y Pastor, 2019). Asimismo, los resultados apuntan a una alta prevalencia de violencia y control en las RRSS (Borrajo y Gámez, 2016; Vázquez y Pastor, 2018). El control, en el cual uno de los miembros de la relación necesita conocer en todo momento las actividades realizadas por su pareja, coincidiendo con otras investigaciones vinculadas a la temática (Lucero, Weisz, Smith-Darden y Lucero, 2014; Borrajo y Gámez, 2016; Vázquez y Pastor, 2019). Además, se logró establecer que terminan por entregar claves de acceso a las distintas plataformas (Rueda, Lindsay y Williams, 2014), siendo coincidente con lo identificado por Martín (2016) quien afirma que existe una presencia de 
suplantación de identidad y violación de intimidad. Mientras que en otro estudio se menciona a los hombres como agresores recurrentes, pero también como víctimas en redes sociales (García et al., 2017).

La violencia física, aunque es la que presenta menor presencia y es la menos normalizada en comparación con otras violencias, el estudio evidencia niveles bajos, aun cuando existe una elevada bidireccionalidad. Por ejemplo, empujar presenta un $12 \%$ de prevalencia para ambos sexos y lanzarse objetos un 7\%. Esto es coincidente con los presentado por Vivanco (2015) quien afirma que las mujeres además, de ser víctimas de la violencia de género también son agresoras en las relaciones de parejas. Si bien la violencia física es la que presenta porcentajes menores en comparación con otras, la violencia sexual también marca unos porcentajes elevados entre ambos sexos (bidireccionalidad). Como se vio en los datos analizados, ceder a los deseos sexuales de sus parejas tiene un $41 \%$ de prevalencia para ambos sexos.

La bidireccionalidad de la violencia en relaciones afectivo-sexuales en jóvenes está en parte explicado por la igualdad de poderes que se establecen en este tipo de relaciones (O’Leary y Slep, 2003; Vizcarra y Póo, 2011; Sanhueza, 2016). De acuerdo con los elementos disponibles en la literatura actual, existirían elementos diferenciadores entre las relaciones de parejas adolescentes y adultos. Las relaciones de adultos están marcadas por la cohabitación, hijos en común y sobre todo la economía familiar. Estos elementos han orientado tanto la legislación, como la investigación a la violencia intrafamiliar. En estas relaciones, es el hombre quien principalmente ejerce la violencia en todas las formas y expresiones: física, psicológica, sexual y económica (De Alencar-Rodriguez y Cantera, 2012). En contraste con lo anterior, la bidireccionalidad identificada en las relaciones adolescentes se explicaría en parte, porque existe un equilibrio de poderes dentro de la relación (Sanhueza, 2017). Es decir, por la inexistencia de la cohabitación. Este factor adquiere importancia puesto que las obligaciones y desigualdades que viven a diario las mujeres en las tareas del hogar, no se aplicarían en estos casos. Por ende, tampoco existe una dependencia económica ya que los jóvenes dependen aún de sus familias de origen. Este equilibro de poder se relacionaría con la bidireccionalidad de la violencia en las relaciones de género. La revisión del estado del arte muestra que la principal causa de la violencia de género contra las mujeres se fundamenta en el patriarcado y en 


\section{Peña Axt \& Sandoval Obando - Violencia de Género}

las desigualdades que esta genera hacia las mujeres (Connell, 2005; Kimmel, 2008). Esta situación es difícil de aplicar en contextos de relaciones de pareja entre adolescentes, puesto que el poder que movilizaría la violencia de género a través del patriarcado, es vivenciado diferencialmente por los adolescentes.

Las masculinidades juegan un papel fundamental en las relaciones de poder y violencia dentro de las relaciones de pareja adolescentes (Connell, 2005; Kimmel 2008). La masculinidad y el género definen la identidad, lo que permite inferir en base a los resultados obtenidos en este estudio, que en la mayoría de las variables son los hombres quienes acusan un leve, pero mayor porcentaje de violencia en las variables que se analizaron en apartado anterior. Al respecto, Flecha et al. (2013) aportan tres tipos de masculinidad: Masculinidad Tradicional Dominante, Nuevas Masculinidades Alternativas y Masculinidad Tradicional Oprimido. Este último modelo de masculinidad, si bien viven en un contexto de patriarcado, son hombres que son víctimas de las desigualdades de este patriarcado, son hombres que tienen relaciones violentas, pero en lugar de ser el hombre quien ejerce esta violencia viven en relaciones de poder, pero inversas. Esto explicaría la cantidad de variables analizadas en las se muestran a los hombres como víctimas por sobre las mujeres.

Lo anteriormente expuesto, estaría relacionado a la Masculinidad Tradicional Dominantes (Flecha et al., 2013) o la Masculinidad Hegemónica (Connell, 2005). En palabras de Connell (2005) refiere al tipo de masculinidad que sostiene el patriarcado y con ello todas las formas de violencia contra las mujeres. Por ende, el sostenimiento de las desigualdades contra las mujeres así como las manifestaciones de violencia están basadas en la heteronormalidad que se ven reflejadas en los datos. Los resultados obtenidos en este trabajo apuntan a un mayor porcentaje de violencia en hombres, visualizándose como una de las causantes de la violencia de género contra las mujeres: la Masculinidad Hegemónica (Connell, 2005).

La violencia forma parte de los procesos de construcción de la masculinidad (Messerschmidt, 2019). La división sexual de la sociedad hace que los hombres sean socializados tempranamente en la normalización de la violencia y la débil promoción de la perspectiva de género (Kimmel, 2008; Ravn, 2018). Lo anterior implica una construcción de la masculinidad que obliga a los hombres a ocultar la violencia de la cual son víctimas, producto 
de los roles que les corresponde cumplir frente a sus pares. La socialización de la masculinidad y el contexto en el que se reproducen relaciones de género violentas (Sommer et al., 2018) que son reflejadas en este estudio: los hombres además de ejercer la violencia la viven y ocultan.

\section{Conclusiones}

Los resultados obtenidos en este estudio apuntan a dos elementos claves, el primero tal y como se ha visto, hace alusión a la bidireccionalidad de la violencia en relaciones adolescentes. En segundo lugar, en gran parte de las variables analizadas, la violencia es mayormente vivenciada por los hombres, siendo coherente con lo reportado por Vivanco et al. (2015) en donde se identificó un mayor ejercicio de esta violencia por parte de las mujeres hacia los hombres. Si bien la literatura nos ofrece luces respecto a la interpretación de estos resultados, lo acotado del estudio impide sacar mayores conclusiones al respecto. Sin embargo, estos resultados reiteran la necesidad de desarrollar futuras investigaciones en las que sea posible profundizar sobre los mecanismos que configuran las dinámicas de violencia de género en adolescentes, puesto que la investigación es escasa y aún más, cuando se aborda la violencia que experimentarían los hombres a lo largo de su vida.

Como parte de las proyecciones que emergen de este trabajo, se podría inferir que las actitudes de rol de género y las dinámicas relacionales que se construyen en la adolescencia, funcionan sinérgicamente para aumentar el riesgo de violencia en el noviazgo. Por esta razón, adquiere valor el desarrollo de estrategias centradas en la modificación de las creencias de los participantes sobre la aceptabilidad de la violencia en el noviazgo, y al mismo tiempo, promoverían actitudes de género más igualitarias y respetuosas entre los más jóvenes (Reyes, et al., 2016). Adicionalmente, avanzar en la implementación de mecanismos preventivos y psicoeducativos en torno la violencia en el noviazgo, particularmente durante la adolescencia temprana, permitiría compensar la posible influencia negativa de las creencias que sostienen los roles tradicionales de género (masculinidad hegemónica o masculinidad tradicional dominante por ejemplo). Es decir, entre los niños con creencias altamente tradicionales, es posible reducir la probabilidad de perpetrar violencia en el noviazgo adolescente, inculcando o fortaleciendo 


\section{Peña Axt \& Sandoval Obando - Violencia de Género}

creencias que rechazan la violencia como un medio de resolución de problemas en las relaciones íntimas fortaleciendo un modelo alternativo de masculinidad o nuevas masculinidades alternativas (Flecha et al., 2013; Ríos et al., 2018). Para ello, es importante la investigación en Nuevas Masculinidades Alternativas que profundicen en el conocimiento de masculinidades que rechazan todo tipo de violencia, no solo la que ejercen hombres, sino que también aquella violencia que pueden estar recibiendo.

Finalmente, es posible señalar que a pesar de que se ha avanzado en la incorporación de la perspectiva de género en la sociedad, promoviendo una mayor valoración de la fuerza laboral femenina, así como la participación masculina en las tareas domésticas, configurando relaciones más igualitarias (Cotter, Hermsen y Vanneman, 2011) en la sociedad es aún insuficiente. Por lo tanto, investigaciones futuras deberían tomar como punto de partida los hallazgos encontrados en este estudio, para avanzar en la exploración y desarrollo de medidas integrales basadas en la perspectiva de género y el desarrollo evolutivo de los sujetos (socialización entre pares, modelado o refuerzo de las masculinidades tradicionales, etc.), para identificar las combinaciones específicas de creencias que influyen en el comportamiento de patrones relacionales violentos a lo largo del ciclo vital, es decir, si las relaciones que detectamos pueden cambiar o no, y cómo pueden cambiar entre la adolescencia y la edad adulta.

\section{Notas}

Los resultados expuestos en este trabajo forman parte del estudio denominado "Violencia de género adolescente en estudiantes de enseñanza media de establecimientos públicos, privados y particulares subvencionados de la ciudad de Temuco" proyecto N. ${ }^{\circ}$ 0141-16. Financiado por la Vicerrectoría de Investigación y Postgrado de la Universidad Autónoma de Chile.

\section{Referencias}

Adams, H.L. y Williams, L. R. (2014). "It's not just you two": A grounded theory of peer-influenced jealousy as a pathway to dating violence among acculturating Mexican American adolescents. Psychology of Violence, 4(3), 294-308. https://psycnet.apa.org/buy/2014-01188-001 Águila Gutiérrez, Yaíma, Hernández Reyes, Vicente Enrique, \& Hernández 
Castro, Vicente Higinio. (2016). Las consecuencias de la violencia de género para la salud y formación de los adolescentes. Revista Médica Electrónica, 38(5), 697-710.

http://scielo.sld.cu/scielo.php?script=sci_arttext\&pid=S1684$18242016000500005 \& \operatorname{lng}=$ es\&tlng=es

Aguirre, A. y García, M. (1997). Violencia prematrimonial: Un estudio exploratorio en universitarios. Última Década (6), 229-245 https://goo.gl/TfxVSh

Albertson, K. M., Moreno, M. A., Garrison, M. M., Evans, Y. N., \& Ahrens, K. R. (2018). Impacts of media on sexual behaviour and relationships among youth in foster care. Child \& Family Social Work, 23(1), 8896. http://doir.org/10.1111/cfs.12387

Bair-Merritt, M. \& Matson, P. (2017). The Dynamic Context of Teen Dating Violence within Adolescent Relationships (Summary Overview).

National Criminal Justice Reference Service.

https://ncvc.dspacedirect.org/handle/20.500.11990/648

Benería, L. (1981). Reproducción, Producción y División Sexual del Trabajo. Mientras Tanto, 6, 47-84.

Bittar, D. B., \& Nakano, A. M. S. (2017). Symbolic violence among adolescents in affective dating relationships. Revista da Escola de Enfermagem da USP, 51. http://doi.org/10.1590/s1980220x2017003003298

Blanco, M. (2014). Implicaciones del uso de las redes sociales en el aumento de la violencia de género en adolescentes. Comunicación y Medios, 30(2014), 124-141. https://goo.gl/KzYZeC

Borrajo, E. \& Gámez-Guadix, M. (2016). Abuso "online" en el noviazgo: relación con depresión, ansiedad y ajuste diádico. Psicología Conductual, 24(2), 221-235. https://goo.gl/Ue1tNv

Canales, M. (2006). Metodología de la Investigació Social. Introducció a los oficios. LOM Edicones. Santiago de Chile.

Connell, R. W. (2005). Masculinities. Cambridge: Polity Press.

Connolly, J., \& McIsaac, C. (2011). Romantic relationships in adolescence.

En M. K. Underwood \& L. H. Rosen (Eds.), Social development:

Relationships in infancy, childhood, and adolescence (pp. 180-203).

Guilford Press. 
Cotter, D.; Hermsen, J. \& Vanneman, R. (2011). End of the gender revolution? Gender role attitudes from 1977 to 2008. American Journal of Sociology, 117, 259-289.

Da Silva, G. P. T. (2016). Possíeveis contribuiçôes das redes sociais mediadas pela internet para os relacionamientos amorosos. Multiverso: Revista Eletrônica do Campus Juiz de Fora-IF Sudeste $M G, 1(2), 181-195$.

http://periodicos.jf.ifsudestemg.edu.br/multiverso/article/view/59

Dávila, O. (2004). Adolescencia y juventud: de las nociones a los abordajes. Última década, 12(21), 83-

104. https://dx.doi.org/10.4067/S0718-22362004000200004

Deans, H., \& Bhogal, M. S. (2019). Perpetrating cyber dating abuse: A brief report on the role of aggression, romantic jealousy and gender.

Current Psychology, 38(5), 1077-1082. http://doi.org/10.1007/s12144017-9715-4

Díaz-Aguado, M (2003). Adolescencia, sexismo y violencia de género. Papeles del Psicólogo, 23(84), 35-44. https://goo.gl/tkzUML

Donoso Vázquez, T., Rubio Hurtado, M., \& Vilà Baños, R. (2017). La Adolescencia ante la violencia de género 2.0: concepciones, conductas y experiencias. Educación XX1, 2l(1).

Duque, E. (2006). Aprendiendo para el amor o para la violencia. Barcelona: El Roure.

Estébanez, I. (2012). Del amor al control a golpe de click: la violencia de género en las redes sociales. IV Jornada de Sensibilización sobre la Violencia de Género. Lanzarote. http://goo.gl/c53TDo

Epstein, M., Furlong, M., Kosterman, R., Bailey, J. A., King, K. M., Vasilenko, S. A., . . Hill, K. G. (2018). Adolescent age of sexual initiation and subsequent adult health outcomes. Am J Public Health, 108(6), 822-828. http://doi.org/10.2105/AJPH.2018.304372

Flecha, R., Puigvert, L., \& Rios, O. (2013). The new alternative masculinities and the overcoming of gender violence. Int. Multidiscip. J. Soc. Sci. 2, 88-113. http://doi.org/10.4471/rimcis.2013.14

Gaete, V. (2015). Desarrollo psicosocial del adolescente. Revista chilena de pediatría, 86(6), 436443. https://dx.doi.org/10.1016/j.rchipe.2015.07.005 
García, P., Guevara, C., Rojas, J., Peña, F. \& González, V. (2017). Apego y ciber-violencia en la pareja de adolescentes. International Journal of Developmental and Educational Psychology, 2(1).

https://goo.gl/E2XDwp

Gómez, J. (2004). El amor en la sociedad del riesgo: una tentativa educativa. Barcelona: El Roure.

Gómez, F. E. H. (2017). Capital social de acceso: desigualdad entre jóvenes a través de sus redes sociales. Revista San Gregorio, 2(17), 56-69.

Gómez-Gamero, M. E., Rodríguez-Hernández, J., \& Quintanar-Vázquez, A. (2019). La Violencia en el noviazgo, los indicios que no se visibilizan. DIVULGARE Boletín Científico de la Escuela Superior de Actopan, 6(12), 32-39.

González-Ortega, I., Echeburúa, E., \& Corral, P. D. (2008). Variables significativas en las relaciones violentas en parejas jóvenes: una revisión. Psicología conductual, 16(2), 207-225.

Hernando-Gómez, A., Maraver-López, P. \& Pazos-Gómez, M. (2016). Experiencias positivas y negativas en relaciones de pareja de jóvenes y adolescentes. Revista de psicología, 25(2), 01-19.

https://goo.gl/SyFfBZ

Instituto Nacional de la Juventud. (2016). Sondeo $N^{\circ} 4$ Violencia en el Pololeo: Jóvenes entre 15 y 29 años. https://goo.gl/ucvSYf Instituto Nacional de la Juventud (2017). Octava Encuesta Nacional de Juventud 2015. https://goo.gl/TNVD9D

Kahn, N. F., \& Halpern, C. T. (2018). Associations between patterns of sexual initiation, sexual partnering, and sexual health outcomes from adolescence to early adulthood. Archives of Sexual Behavior, 47(6), 1791-1810. http://doi.org/10.1007/s10508-018-1176-9

Kaufman-Parks, A., DeMaris, A., Giordano, P. C., Manning, W. D., \& Longmore, M. A. (2018). Familial effects on intimate partner violence perpetration across adolescence and young adulthood. Journal of Family Issues, 39(7), 1933-1961. doi:10.1177/0192513X17734586 Kimmel, M. (2008). Los estudios de la masculinidad: una introducción. En Carabí, A. \& Armengol, M. (Ed.) La masculinidad a debate. Icaria. Lehrer, J., Lehrer, E. \& Oyarzún, P. (2009). Violencia sexual en hombres y mujeres jóvenes en Chile: Resultados de una encuesta (año 2005) a 


\section{Peña Axt \& Sandoval Obando - Violencia de Género}

estudiantes universitarios. Revista médica de Chile, 137(5), 599608. https://dx.doi.org/10.4067/S0034-98872009000500002

Linne, J. W., \& Angilletta, M. F. (2016). Violencia en la red social: una indagación de expresiones online en adolescentes de sectores populares marginalizados del Área Metropolitana de Buenos Aires. Salud colectiva, 12, 279-294.

Lucero, J.; Weisz, A.; Smith-Darden, J. \& Lucero, S. (2014). Exploring gender differences: Socially interactive technology use/abuse among dating teens. Affilia, 29(4), 478-491.

Mena-Rodriguez, E., \& Velasco-Martínez, L. (2017). Gender violence and social networks in adolescents. the case of the province of Malaga. Procedia-Social and Behavioral Sciences, 237, 44-49.

https://doi.org/10.1016/j.sbspro.2017.02.009

Martín, A., Pazos, M., Montilla, M. \& Romero, C. (2016). Una modalidad actual de violencia de género en parejas de jóvenes: Las redes sociales. Educación XX1, 19(2), 405-429. https://dx.doi.org/10.5944/educxx1.16473

Martínez-Marín, M. D., \& Martínez, C. (2019). Negative and positive attributes of gender stereotypes and gender self-attributions: A study with spanish adolescents. Child Indicators Research, 12(3), 10431063. http://doi.org/10.1007/s12187-018-9569-9

Messerschmidt, J. (2019). Nine lives: Adolescent masculinities, the body and violence. Routledge.

Messinger, A. M. (2018). Bidirectional same-gender and sexual minority intimate partner violence. Violence and Gender, 5(4), 241-249. http://doi.org/10.1089/vio.2018.0001

Mingo, A., \& Moreno, H. (2015). El ocioso intento de tapar el sol con un dedo: violencia de género en la universidad. Perfiles educativos, 37(148), 138-155.

Mrug, S. \& Windle, M. (2009). Bidirectional influences of violence exposure and adjustment in early adolescence: Externalizing behaviors and school connectedness. Journal of Abnormal Child Psychology, 37(5), 611-623. http://doi.org/10.1007/s10802-009-93046

Novo, M., Herbón, J., \& Amado, B. (2016). Género y victimización: Efectos 
en la evaluación de la violencia psicológica sutil y manifiesta, apego adulto y tácticas de resolución de conflictos. Revista Iberoamericana de Psicología y Salud, 7, 89-97. https://goo.gl/67P525

O’Leary, K. \& Slep, A. (2003). A Dyadic Longitudinal Model Of Adolescent Dating Aggression. Journal of clinical child and adolescent psychology, 32(3), 314-327. https://goo.gl/54v8eg Padrós, M. (2012). Modelos de Atractivo Masculinos en la Adolescencia. Masculinidades y cambio social, 1(2), 165-183.

Palmetto, N.; Davidson, L. L. \& Rickert, V. (2013). Predictors of physical intimate partner violence in the lives of young women: Victimization, perpetration, and bidirectional violence. Violence and Victims, (1), 103-121 http://doi.org/10.1891/0886-6708.28.1.103

Peña J. C., Arias, L. L., \& Boll, V. (2019). Los celos como norma emocional en las dinámicas de violencia de género en redes sociales en las relaciones de pareja de estudiantes de Temuco, Chile. Multidisciplinary Journal of Gender Studies, 8(2), 180-203. http://dx.doi.org/10.17583/generos.2019.4223

Ravn, S. (2018). "I Would Never Start a Fight but...” Young Masculinities, Perceptions of Violence, and Symbolic Boundary Work in Focus Groups. Men and masculinities, 21(2), 291-309.

Rey-Anacona, C; (2013). Prevalencia y tipos de maltrato en el noviazgo en adolescentes y adultos jóvenes. Terapia Psicológica, 31(2), 143-154. https://goo.gl/WDa9xq

Reyes, H. L., Foshee, V. A., Niolon, P. H., Reidy, D. E., \& Hall, J. E. (2016). Gender role attitudes and male adolescent dating violence perpetration: Normative beliefs as moderators. Journal of Youth and Adolescence, 45(2), 350-360. http://doi.org/10.1007/s10964-0150278-0

Rios-González, O., Peña Axt, J. C., Duque Sánchez, E., \& De Botton Fernández, L. (2018). The language of ethics and double standards in the affective and sexual socialization of youth. Communicative acts in the family environment as protective or risk factors of intimate partner violence. Frontiers in Sociology, 3, 19. https://doi.org/10.3389/fsoc.2018.00019

Rueda, H., Lindsay, M., \& Williams, L. (2014). "She posted it on 


\section{Peña Axt \& Sandoval Obando - Violencia de Género}

Facebook": Mexican American adolescents' experiences with technology and romantic relationship conflict. Journal of Adolescent Research, 30(4), 419-445.

Saldivia, C \& Vizcarra, B. (2012). Consumo de Drogas y Violencia en el Noviazgo en Estudiantes Universitarios del Sur de Chile. Terapia Psicológica, 30(2). 43-49 https://goo.gl/5UiT5L

Sandoval, E. \& Peña, J.C. (2019). Violencia de Género y discriminación arbitraria en una universidad privada chilena: una exploración inicial. Sophia, 15(2); 55-70. https://doi.org/10.18634/sophiaj.15v.2i.945

Sandoval, E. (2018). Nudos Críticos en torno a la Educación No Sexista:

Una Deconstrucción Necesaria para Todxs. Otras Voces en Educación. http://otrasvoceseneducacion.org/archivos/281088

Sanhueza, T. (2016). Violencia en las relaciones amorosas y violencia conyugal: Convergencias y divergencias. Reflexiones para un debate. Última década, 24(44), 133167. https://dx.doi.org/10.4067/S0718-22362016000100006

Schuster, I., Krahé, B., Ilabaca, P. \& Muñoz-Reyes, J. (2016). Sexual Aggression Victimization and Perpetration among Male and Female College Students in Chile. Frontiers in Psychology. 7(1354). https://goo.gl/DbsdjD

Sommer, M.; Muñoz-Laboy, M.; Williams, A.; Mayevskaya, Y.; Falb, K.; Abdella, G. y Stark, L. (2018). How gender norms are reinforced through violence against adolescent girls in two conflict-affected populations. Child Abuse \& Neglect, 79, 154-163.

https://doi.org/10.1016/j.chiabu.2018.02.002

Valdivia-Peralta, M., Fonseca-Pedrero, E., González Bravo, L., \& Paíno Piñeiro, M. (2019). Invisibilización de la violencia en el noviazgo en Chile: evidencia desde la investigación empírica. Perfiles latinoamericanos, 27(54).

https://perfilesla.flacso.edu.mx/index.php/perfilesla/article/view/782

Vázquez, N., \& Pastor, Y. (2019). Uso de redes sociales y mensajería instantánea en relaciones de pareja en la juventud: Un estudio preliminar. Health \& Addictions/Salud y Drogas, 19(1).

Villanueva-Blasco, V. \& Grau-Alberola, E. (2019). Gender and Age Differences in the Internalization of Gender Stereotypes in Early and 
Mid Adolescence. Electronic Journal of Research in Educational Psychology, 17(47), 107-128.

http://ojs.ual.es/ojs/index.php/EJREP/article/viewFile/2184/2838

Vivanco, R., Espinoza, S., Romo, C., Véliz, A. \& Vargas, A. (2015).

Perpetración y victimización de la violencia en relaciones de parejas en jóvenes que cursan educación superior en la ciudad de Osorno, Chile. Polis (Santiago), 14(40), 489-

508. https://dx.doi.org/10.4067/S0718-65682015000100023

Vizcarra, B; Póo, A. (2011). Violencia de pareja en estudiantes universitarios del sur de Chile. Universitas Psychologica, 10(1), 8998. https://goo.gl/Tst31W

Wright, M. F. (2020). The role of technologies, behaviors, gender, and gender stereotype traits in adolescents' cyber aggression. $J$ Interpers Violence, 35(7-8), 1719-1738.

http://doi.org/10.1177/0886260517696858

Xóchitl, I., Sánchez, A. \& Robles, F. (2013). Relaciones entre estilos de amor y violencia en adolescentes. Psicología Desde El Caribe, 30(2) https://goo.g1/MFU32o

Juan Carlos peña Axt is a Professor at the Universidad Autónoma de Chile

Eduardo Sandoval-Obando i is a Professor at the Universidad Autónoma de Chile

Contact Address: juan.pena@uautonoma.cl 